\title{
TERAPIA FONOLÓGICA: A GENERALIZAÇÃO PARA OUTRA POSIÇÃO NA PALAVRA
}

\section{Phonological therapy: the generalization to other position in the word}

\author{
Marizete Ilha Ceron ${ }^{(1)}$, Márcia Keske-Soares ${ }^{(2)}$
}

\begin{abstract}
RESUMO
Objetivo: analisar a eficácia de três modelos de terapia com base fonológica em sujeitos com diferentes gravidades do desvio fonológico. Métodos: o grupo pesquisado foi constituído de 21 crianças, 11 do sexo masculino e dez do feminino, com média de idade de 5:7 anos. Os dados da fala dos sujeitos foram analisados por meio da avaliação fonológica. Após a realização da análise contrastiva, foi calculado o Percentual de Consoantes Corretas proposto por Shriberg e Kwiatkowski (1982) e os sujeitos foram classificados em diferentes gravidades do desvio fonológico em desvio severo, moderado-severo, médio-moderado e médio. Sendo seis sujeitos submetidos ao Modelo de Ciclos Modificado, oito ao Modelo ABAB-Retirada e Provas Múltiplas, e sete ao Modelo Oposições Máximas Modificado. Fez-se uma comparação da generalização para outras posições da palavra entre os três modelos de terapia em cada gravidade. Resultados: a generalização a outras posições na palavra ocorreu em todos os modelos estudados, todavia, os maiores percentuais de acertos obtidos foram no Modelo ABAB-Retirada e Provas Múltiplas, no grupo com desvio severo; no Modelo Oposições Máximas Modificado, nos grupos com desvio moderado-severo e médio-moderado; e nos Modelos Oposições Máximas Modificado e ABAB-Retirada e Provas Múltiplas, no grupo com desvio médio. Conclusão: todos os sujeitos apresentaram evoluções no sistema fonológico e quanto à generalização para outras posições na palavra, sendo em maior percentual nos modelos ABAB-Retirada e Provas Múltiplas e Oposições Máximas Modificado.
\end{abstract}

DESCRITORES: Fonoterapia; Generalização; Fala; Distúrbios da Fala

\section{INTRODUÇÃO}

Crianças e adultos se comunicam, principalmente pela linguagem oral, sendo esta constituída por aspectos fonológicos, morfológicos, sintáticos, semânticos e pragmáticos. $\mathrm{O}$ aspecto fonológico ${ }^{1}$ é um dos mais importantes porque constitui o sistema contrastivo da língua, marcando as mudanças de significados entre as palavras.

A aquisição do sistema fonológico ocorre gradativamente até os cinco anos de idade, porém, algu-

(1) Fonoaudióloga; Especialização em Fonoaudiologia pela Universidade Federal de Santa Maria; Mestranda em Distúrbios da Comunicação Humana pela Universidade Federal de Santa Maria.

(2) Fonoaudióloga; Professora do Curso de Fonoaudiologia e do Programa de Pós-Graduação em Distúrbios da Comunicação Humana da Universidade Federal de Santa Maria, UFSM, Santa Maria, RS; Doutora em Linguística Aplicada pela Pontifícia Universidade Católica do Rio Grande do Sul.

Conflito de interesse: INEXISTENTE mas crianças apresentam dificuldades para adquirir o sistema de sons da sua língua, mesmo sem qualquer comprometimento orgânico ${ }^{2}$.

Essa dificuldade de fala, em que algumas crianças não obtem os padrões de fala esperados para sua idade, é denominada por alguns autores ${ }^{3,4}$ de distúrbio fonológico e, por outros ${ }^{5-8}$, de desvio fonológico (DF).

A terapia com base fonológica busca a reorganização do sistema de sons ${ }^{9-11}$ da criança, tendo como princípio básico a generalização ${ }^{11}$. Dentre os modelos de terapia com base fonológica mais utilizados destacam-se: o de Ciclos Modificado 12; o ABAB-Retirada e Provas Múltiplas ${ }^{13}$; e o de Oposições Máximas Modificado ${ }^{14}$.

O Modelo de Ciclos Modificado é composto de ciclos de terapia, sendo que, em cada ciclo, três processos alvos são trabalhados, através de dois sons-alvo. O objetivo principal é facilitar a emergência de padrões fonológicos ao invés de "treinar" segmentos fonêmicos ${ }^{15}$. 
No Modelo ABAB-Retirada e Provas Múltiplas um som-alvo é estimulado durante 5 semanas (9 sessões), a escolha desses alvos estão em conformidade com a hierarquia implicacional de traços. A partir da análise fonológica do sistema da criança é possível planejar os alvos a serem tratados, tendo em vista que ensinar sons mais complexos resultaria em maiores mudanças no sistema de sons da criança, facilitando a aquisição não só do som-alvo ${ }^{15}$.

O Modelo de Oposições Máximas Modificado ${ }^{14}$ tem como propósito fazer com que a criança reorganize seu sistema fonológico através da percepção auditiva, da imitação e produção espontânea das palavras-alvo. Na primeira etapa, deve imitar o modelo dado pelo terapeuta e, atingir um percentual de $80 \%$ ou mais de produções corretas para poder passar para a etapa seguinte, de produção espontânea. Na segunda etapa, os alvos devem ser produzidos sem o modelo imediato do terapeuta.

Como referido acima, a possibilidade de favorecer a generalização é a principal contribuição dos modelos fonológicos ${ }^{11,16,17}$, pois a ocorrência desta leva a uma terapia mais eficiente, não precisando ensinar todos os sons incorretos em todas as palavras e em diferentes posições dentro das palavras ${ }^{16}$. A presença de generalização foi relatada no tratamento de sujeitos com DF em muitos estudos internacionais ${ }^{18-21}$ e nacionais ${ }^{7,8,16,22-25}$.

A generalização é definida como a ampliação da produção e uso correto dos sons-alvo treinados em outros contextos ou ambientes não-treinados, podendo ser analisada sob dois enfoques: o funcional, modo pelo qual cada criança reorganiza seu sistema de sons; e o estrutural, refere-se à identificação das circunstâncias sob as quais ela ocorre ${ }^{26}$.

Em relação aos componentes estruturais, a generalização para outras posições na palavra refere-se ao caso da criança aprender um somalvo numa determinada posição e realizar este som corretamente em outra posição, sem tratamento direto ${ }^{15,16}$.

Apesar disso, são poucos os estudos ${ }^{16,24}$ conhecidos que comparam a generalização para outras posições na palavra obtida em diferentes modelos de tratamento, sendo necessários novos trabalhos, pois essas pesquisas podem auxiliar o clínico na escolha de modelos de terapia mais eficazes para cada caso.

Portanto, o objetivo deste estudo é analisar a eficácia de três modelos de terapia com base fonológica em diferentes gravidades do DF.

\section{MÉTODOS}

O grupo de sujeitos foi constituído por 21 crianças com DF, 11 do sexo masculino e 10 do feminino, cuja média de idade no início do tratamento era de 5:7 anos. Para que os sujeitos participassem da pesquisa, os pais ou responsáveis assinaram um Termo de Consentimento Livre e Esclarecido autorizando a pesquisa e posterior publicação dos resultados.

Antes do processo terapêutico os sujeitos foram submetidos a diversas avaliações fonoaudiológicas, sendo fundamental, não apresentar alterações significativas nessas avaliações, à exceção da avaliação fonológica. Foram também realizadas avaliações complementares: otorrinolaringológica, audiológica e neurológica, a fim de descartar quaisquer fatores que pudessem interferir no desenvolvimento da linguagem.

Para a coleta dos dados da fala foi aplicada a avaliação fonológica da criança (AFC) ${ }^{27}$ antes e após a terapia, sendo realizada pela nomeação de figuras que continham todos os sons constrastivos do português em todas as posições da sílaba e da palavra. Para análise dos dados da fala utilizou-se a ficha de análise contrastiva. Com base nesta análise foi possível obter o sistema fonológico de cada sujeito.

$\mathrm{Na}$ obtenção do sistema fonológico, um segmento foi considerado adquirido quando ocorreu de $80 \%$ a $100 \%$ das vezes, parcialmente adquirido quando ocorreu de $40 \%$ - $79 \%$, e não adquirido quando ocorre de $0 \%-39 \%$ das possibilidades ${ }^{28}$.

Os sujeitos foram divididos em três grupos de acordo com o modelo de terapia fonológica e cada grupo foi tratado com um modelo diferente, sendo seis (C3 ao C8) submetidos ao tratamento pelo Modelo de Ciclos Modificado; oito (A1 ao A8) pelo ABAB-Retirada e Provas Múltiplas; e sete (O1 ao O8) ao de Oposições Máximas Modificado.

Em cada modelo terapêutico, os sujeitos foram classificados no início do tratamento em diferentes gravidades do DF, a partir do cálculo do Percentual de Consoantes Corretas (PCC) ${ }^{29}$. Os sujeitos foram classificados em desvio severo (A1, A2, 01 e O2), com PCC menor que $50 \%$; desvio moderado-severo (C3, C4, A3, A4 e O3), com PCC de 51 a 65\%; desvio médio-moderado ( $\mathrm{C} 5, \mathrm{C} 6, \mathrm{~A} 5, \mathrm{~A} 6, \mathrm{O} 5$ e O6), com PCC entre 66 e $85 \%$; e desvio médio ( $C 7, C 8$, A7, A8, O7 e O8), com PCC de 86 a 100\%.

Este estudo está vinculado ao projeto de pesquisa aprovado pelo Comitê de Ética em Pesquisa (CEP) de uma instituição de ensino superior sob $n^{-}$ 046/02. 
A fim de verificar a eficácia dos três modelos de terapia, analisou-se e descreveu-se qualitativamente a generalização para outras posições na palavra. Para constatar esta generalização e comparar entre os modelos terapêuticos em diferentes gravidades do DF examinou-se o aumento do percentual de acertos entre a avaliação fonológica inicial e final de cada sujeito.

\section{RESULTADOS}

Na Tabela 1 estão apresentados os resultados da generalização para outras posições na palavra verificadas nos sujeitos com desvio severo e moderado-severo tratados pelos três diferentes modelos de terapia (Modelo de Ciclos Modificado, Modelo ABAB-Retirada e Provas Múltiplas, Modelo Oposições Máximas Modificado).

Tabela 1 - Generalização para outras posições na palavra nos sujeitos com desvio severo e moderado-severo

\begin{tabular}{|c|c|c|c|c|}
\hline \multirow{2}{*}{ Sujeito } & \multirow{2}{*}{$\begin{array}{l}\text { Som-alvo } \\
\text { Posição }\end{array}$} & \multirow{2}{*}{ Outras posições } & \multicolumn{2}{|c|}{$\%$ de acertos } \\
\hline & & & $\mathrm{Al}$ & AF \\
\hline \multirow{2}{*}{ A1 } & [d3] Ol e OM & * & * & $*$ \\
\hline & /l/ Ol e OM & * & * & * \\
\hline \multirow{4}{*}{ A2 } & IK/ OM & * & * & * \\
\hline & $/ r / O M$ & CM & 0,00 & 80,64 \\
\hline & & $\mathrm{CF}$ & 0,00 & 100,00 \\
\hline & $13 / 01$ & OM & 0,00 & 75,86 \\
\hline \multirow{3}{*}{01} & $/ \mathrm{s} / \mathrm{OM}$ & $\mathrm{OI}$ & 0,00 & 42,85 \\
\hline & & $\mathrm{CM}$ & 0,00 & 20,00 \\
\hline & & $\mathrm{CF}$ & 0,00 & 0,00 \\
\hline \multirow{3}{*}{$\mathbf{O 2}$} & /z/ OM & $\mathrm{Ol}$ & 0,00 & 100,00 \\
\hline & $/ r / O M$ & $\mathrm{CF}$ & 0,00 & 50,00 \\
\hline & & $\mathrm{CM}$ & 0,00 & 0,00 \\
\hline \multirow{3}{*}{ C3 } & $\mathrm{lg} / \mathrm{OI}$ e OM & * & * & * \\
\hline & /3/ OI e OM & * & * & * \\
\hline & /R/ OI e OM & & & \\
\hline \multirow{7}{*}{$\mathrm{C} 4$} & /s/ Ol e OM & CM & 0,00 & 0,00 \\
\hline & |z/ Ol e OM & * & * & * \\
\hline & /I/ Ol e OM & * & * & * \\
\hline & & * & * & * \\
\hline & th/ & OM & 100,00 & 100,00 \\
\hline & /R/OI & $\mathrm{CM}$ & 16,67 & 7,69 \\
\hline & Ir/ OIVI & $\mathrm{CF}$ & 0,00 & 0,00 \\
\hline \multirow{3}{*}{ A3 } & $\mathrm{lg} / \mathrm{OI}$ & OM & 0,00 & 23,91 \\
\hline & $/ \mathrm{r} / \mathrm{OM}$ & $\mathrm{CM}$ & 0,00 & 91,23 \\
\hline & & $\mathrm{CF}$ & 0,00 & 95,00 \\
\hline \multirow{5}{*}{ A4 } & /R/ OI e OM & $*$ & * & * \\
\hline & /N/ OM & * & * & * \\
\hline & $/ \mathrm{r} / \mathrm{OM}$ & $\mathrm{CM}$ & 0,00 & 38,89 \\
\hline & $1110 \mathrm{TV}$ & $\mathrm{CF}$ & 0,00 & 85,71 \\
\hline & $/ \mathrm{r} / \mathrm{CM}$ e CF & OM & 4,26 & 91,67 \\
\hline \multirow{2}{*}{ O3 } & /R/ OI & OM & 0,00 & 100,00 \\
\hline & /l/ OI & OM & 0,00 & 80,00 \\
\hline
\end{tabular}

Legenda: Al: avaliação inicial. AF: avaliação final. Ol: onset inicial. OM: onset medial. CM: coda medial. CF: coda final. * não houve possibilidade de ocorrência devido à escolha da posição do som-alvo. s/o: sem ocorrência 
Os alofones [t]], [d3] serão referenciados entre colchetes, para diferenciá-los dos demais fonemas, uma vez que não são fones contrastivos no Português.

Comparando a generalização a outras posições na palavra entre os diferentes modelos aplicados, verificou-se que, no grupo com desvio severo, o sujeito que mais generalizou a outras posições na palavra foi o tratado pelo Modelo ABAB-Retirada e Provas Múltiplas e Oposições Máximas Modificado (o A2, seguido do O2). No grupo com desvio moderado-severo, foram $\mathrm{O} 3$, seguido do $\mathrm{A} 4$ e $\mathrm{A} 3$ submetidos, respectivamente, aos Modelos Oposições Máximas Modificado e ABAB-Retirada e Provas Múltiplas.

$\mathrm{Na}$ Tabela 2 são apresentados os resultados da generalização para outras posições na palavra veri- ficadas nos sujeitos com desvio médio-moderado e médio tratados pelos modelos: Ciclos Modificado, ABAB-Retirada e Provas Múltiplas e Oposições Máximas Modificado.

Observando a generalização, constataram-se que, no grupo com desvio médio-moderado, foram O5, seguido do O6, tratados pelo Modelo Oposições Máximas Modificado os que mais generalizaram a outras posições. No grupo com desvio médio, foram - 08 e A8, seguido do A7, submetidos aos Modelos Oposições Máximas Modificado e ABAB-Retirada e Provas Múltiplas.

\section{DISCUSSÃO}

Analisando-se a Tabela 1, pode-se observar, no grupo com desvio severo, para o A1 tratado com os

Tabela 2 - Generalização para outras posições na palavra nos sujeitos com desvio médio-moderado e médio

\begin{tabular}{|c|c|c|c|c|}
\hline \multirow{2}{*}{ Sujeito } & \multirow{2}{*}{$\begin{array}{l}\text { Som-alvo } \\
\text { Posição }\end{array}$} & \multirow{2}{*}{ Outras posições } & \multicolumn{2}{|c|}{$\%$ de acertos } \\
\hline & & & Al & AF \\
\hline \multirow{2}{*}{ C5 } & /g/ Ol e OM & * & * & * \\
\hline & /r/ OM e CM & $\mathrm{CF}$ & 100,00 & 57,14 \\
\hline \multirow{3}{*}{ C6 } & $\mathrm{lg} / \mathrm{Ol}$ e OM & * & * & * \\
\hline & /3/ OI e OM & * & * & * \\
\hline & /r/ OM e CF & $\mathrm{CM}$ & 0,00 & 28,57 \\
\hline A5 & /3/ OI e OM & * & * & * \\
\hline A6 & /R/ OI e OM & $*$ & * & * \\
\hline 05 & /z/ OM & Ol & 50,00 & 100,00 \\
\hline \multirow{2}{*}{06} & /g/ Ol & OM & 28,57 & 50,00 \\
\hline & /3/ Ol & OM & 0,00 & 83,33 \\
\hline \multirow[t]{2}{*}{$\mathrm{C7}$} & $/ \mathrm{r} / \mathrm{OM}$ e CM & $\mathrm{CF}$ & 66,67 & 100,00 \\
\hline & /f/ Ol & OM & 66,67 & 100,00 \\
\hline \multirow[t]{2}{*}{ C8 } & $/ \mathrm{r} / \mathrm{OM}$ & $\mathrm{CM}$ & 75,00 & 88,23 \\
\hline & & $\mathrm{CF}$ & 100,00 & 100,00 \\
\hline \multirow{3}{*}{ A7 } & /3/ Ol & OM & 14,29 & 81,82 \\
\hline & $/ \mathrm{r} / \mathrm{CM}$ & OM & 13,72 & 86,93 \\
\hline & & CF & 57,14 & 73,34 \\
\hline \multirow{4}{*}{ A8 } & $/ \mathrm{r} / \mathrm{OM}$ & $\mathrm{CM}$ & 20,00 & 100,00 \\
\hline & & CF & 33,33 & 100,00 \\
\hline & $/ \mathrm{r} / \mathrm{CM}$ & OM & 4,17 & 100,00 \\
\hline & & $\mathrm{CF}$ & 33,33 & 100,00 \\
\hline \multirow{3}{*}{07} & $/ \mathrm{n} / \mathrm{OM}$ & $\mathrm{Ol}$ & 100,00 & 100,00 \\
\hline & /r/ OM & $\mathrm{CM}$ & 54,54 & 100,00 \\
\hline & & CF & 100,00 & 66,66 \\
\hline 08 & [t]] OM & Ol & S/o & 100,00 \\
\hline
\end{tabular}

Legenda: Al: avaliação inicial. AF: avaliação final. OI: onset inicial. OM: onset medial. CM: coda medial. CF: coda final. * não houve possibilidade de ocorrência devido à escolha da posição do som-alvo. s/o: sem ocorrência 
alvos [dz] e /// em OI e OM, não houve possibilidades de ocorrer este tipo de generalização devido à posição dos alvos tratados. Da mesma forma, para o A2, no alvo /N/ em OM. A autora deste estudo ${ }^{23}$ referiu que esse sujeito foi o que apresentou um maior percentual de produções corretas dos alvos em outras posições, quando comparado com os demais sujeitos, pois apresentou um aumento de acertos para o / r/ em CF e CM; e para o/z/ em OM.

Este tipo de generalização também foi constatado para o 01 e 02 . O 02 foi estimulado na posição de OM com o alvo /z/, o qual aumentou as produções corretas em OI. O O2 também generalizou o alvo /r/ para a posição de CF. O O1, estimulado $\mathrm{com} / \mathrm{s} / \mathrm{e} / \mathrm{N} / \mathrm{em} \mathrm{OM}$, apresentou produções corretas para o /s/ em Ol e CM. Outros estudos ${ }^{16,22-25}$ também verificaram este tipo de generalização nos sujeitos analisados.

Corroborando os resultados encontrados para o A2 e O2, quanto à generalização do /r/, um estudo ${ }^{16}$ referiu ter encontrado esse tipo de generalização em dois sujeitos submetidos a diferentes modelos de terapia fonológica (Modelo de Ciclos Modificado e Modelo de Oposições Máximas). O alvo /r/, estimulado em OM, foi adquirido em CM e CF, e o alvo /S/ estimulado em OM determinou o aparecimento deste em Ol. Ambos modelos estudados foram eficazes em relação à reorganização fonológica e promoção de generalizações.

Observou-se que, de todos os sujeitos com desvio severo, o que mais generalizou a outras posições na palavra foi o A2, seguido do O2, tratados respectivamente pelos Modelos ABAB-Retirada e Provas Múltiplas e Oposições Máximas Modificado. A autora do estudo ${ }^{23}$ que utilizou esses sujeitos submetidos ao Modelo ABAB-Retirada e Provas Múltiplas constatou que o grupo com desvio severo foi o que apresentou um maior número de generalização para outras posições na palavra.

Dos sujeitos com desvio moderado-severo, verifica-se que apenas o sujeito C4 não apresentou generalização para outras posições nas palavras em todos os sons-alvo possíveis. Ao contrário dos demais sujeitos pesquisados que generalizaram para as outras posições, conforme as possibilidades de ocorrência. Ao contrário de um estudo ${ }^{17}$ que relatou presença desta generalização em todos os sujeitos pesquisados. A autora ${ }^{23}$ que estudou os dados de fala dos sujeitos submetidos ao modelo ABAB-Retirada e Provas Múltiplas afirmou que os grupos com desvio severo e moderado-severo apresentaram maior número de generalizações para outra posição na palavra.

Para o sujeito A4, ao afirmar que ocorreu a generalização para a posição de OM, quando esti- mulada com /r/ em CM e CF, deve-se considerar o efeito cumulativo da estimulação anterior do $/ \mathrm{r} / \mathrm{em}$ OM. Como já comentado para os sujeitos A2 e O2, uma pesquisa ${ }^{16}$ referiu ter encontrado este tipo de generalização para o alvo /r/. Assim como, outro estudo ${ }^{24}$ da generalização obtida por quatro sujeitos com DF tratados com os róticos / $r /$ e /R/ (dois pelo Modelo ABAB-Retirada e Provas Múltiplas e dois pelo Modelo Oposições Máximas Modificado), relatou que a generalização para outra posição na palavra foi apresentada pelos dois sujeitos tratados com o/r/, sendo que foram aplicados modelos diferentes para cada um.

Este tipo de generalização também foi obtido, pelo O3: quando tratado com o /R/ em OI, generalizou para $\mathrm{OM}$ e, quando estimulado com a líquida I//, também em OI, aumentou as produções corretas em OM. Um estudo ${ }^{24}$ não encontrou esse tipo de generalização nos dois sujeitos tratados com o rótico /R/, contrariando o resultado encontrado para o O3 quando estimulado com o /R/ em Ol.

Observou-se que, de todos os sujeitos com desvio moderado-severo, o que mais generalizou a outras posições foi o O3, seguido do A4 e A3, tratados, respectivamente, pelos Modelos Oposições Máximas Modificado e ABAB-Retirada e Provas Múltiplas. As autoras ${ }^{23,25}$ que utilizaram esses sujeitos em suas pesquisas constataram que 0 grupo com desvio moderado-severo foi o que mais apresentou generalização para outra posição da palavra, em sujeitos tratados pelos Modelos ABABRetirada e Provas Múltiplas e Oposições Máximas Modificado, respectivamente.

Analisando-se o grupo com desvio médio-moderado (Tabela 2), pode-se verificar que apenas 0 sujeito C5 não apresentou generalização para outras posições nas palavras, mesmo quando possível. Ao contrário dos demais sujeitos pesquisados que generalizaram para as outras posições quando tinham possibilidades de ocorrência. Contrariando alguns estudos ${ }^{16,25}$ que relataram a presença desta generalização em todos os sujeitos pesquisados.

Dos sujeitos tratados pelo Modelo de Ciclos Modificado, como já foi mencionado, o C5 não generalizou, enquanto que o C6 teve possibilidade de generalizar apenas para o alvo /r/ em CM. Os sonsalvo /g/ e /3/ não permitiram a ocorrência deste tipo de generalização, devido às posições trabalhadas em terapia. A pesquisa ${ }^{22}$, que utilizou esses sujeitos submetidos ao Modelo de Ciclos Modificado com diferentes gravidades do DF, constatou um maior número de fonemas adquiridos no sistema fonológico final no grupo com desvio médio-moderado, seguido do com desvio médio e, por último, do com desvio moderado-severo. 
Da mesma forma os sujeitos que receberam atendimento pelo Modelo ABAB-Retirada e Provas Múltiplas, A5, estimulado com /3/ em Ol e OM, e A6, tratado com /R/ em Ol e OM, não tiveram possibilidade de generalizar a outras posições devido à escolha das posições a serem trabalhadas durante as sessões de terapia. Ao analisar a generalização para outras posições na palavra obtida pelo Modelo ABAB-Retirada e Provas Múltiplas em 8 sujeitos em quatro grupos de gravidade do DF, a autora ${ }^{23}$ não encontrou esta generalização no grupo com desvio médio-moderado.

Nos demais sujeitos com desvio médio-moderado, verificaram-se este tipo de generalização. $O$ O5 foi o que apresentou um maior percentual de produções corretas: para o alvo/z/, estimulado em OM, aumentou o percentual de acertos em OI. A escolha dos alvos para o 06 permitiu a ocorrência de generalização de $/ \mathrm{g} / \mathrm{e} / 3 /$ em OM. O estudo ${ }^{17}$ referiu ter obtido a generalização a outras posições na palavra em quatros sujeitos que receberam tratamento pelo Modelo Oposições Máximas Modificado.

Observou-se que, de todos os sujeitos com desvio médio-moderado, que tinham a possibilidade de ocorrência da generalização a outras posições na palavra, o 05 foi o que apresentou maiores índices de aumento de produções corretas, seguido do 06. Um estudo ${ }^{25}$ encontrou este tipo de generalização em sujeitos submetido ao Modelo Oposições Máximas Modificado.

No grupo com desvio médio, observou-se que o único que não apresentou generalização para outras posições nas palavras foi o sujeito $\mathrm{O} 7$ para o / $r /$ em CF, ao contrário, dos demais pesquisados que generalizaram. Ambos sujeitos tratados pelo Modelo de Ciclos Modificado generalizaram a outras posições na palavra. O C7 produziu o alvo /r/ corretamente na posição de CF, enquanto que o C8 generalizou o/r/ para a posição de CM e o/f/ para a posição de OM. Os sujeitos que foram submetidos ao tratamento pelo Modelo ABAB-Retirada e Provas Múltiplas generalizaram. O A7 generalizou para /3/ em OM e para /r/ em OM e CF. Para o sujeito A8, ao afirmar que ocorreu a generalização para a posição de $\mathrm{OM}$, quando estimulada com $/ \mathrm{r} / \mathrm{em} \mathrm{CM}$, deve-se considerar também o efeito cumulativo da estimulação do /r/ em OM, já que essa posição foi trabalhada anteriormente. Confirmando os achados de $\mathrm{C} 7, \mathrm{C} 8, \mathrm{~A} 7, \mathrm{~A} 8$, e 07, um estudo ${ }^{24}$ relatou esse tipo de generalização em sujeitos tratados com o rótico $/ \mathrm{r} /$.

O O7, tratado pelo Modelo Oposições Máximas Modificado, como já foi comentado, não generalizou para o/r/ para a CF, mas para a CM apresentou um aumento de produções corretas. Para o O8, houve generalização de [t $\mathrm{t}]$ para OI.

Observou-se que de todos os sujeitos com desvio médio, os que mais generalizaram a outras posições não trabalhadas em terapia foram o 08 e $A 8$, seguido do $A 7$, tratados respectivamente pelos Modelos Oposições Máximas Modificado e ABAB-Retirada e Provas Múltiplas. Alguns estudos 17,21,22,24,25 referem a ocorrência desse tipo de generalização.

\section{CONCLUSÃO}

Com base nos resultados desta pesquisa verificou-se que a maioria dos sujeitos nas diversas gravidades acrescentou fonemas nos seus sistemas fonológicos, sendo os que adquiriram mais fonemas no pós-tratamento foram os sujeitos tratados pelo Modelo ABAB-Retirada e Provas Múltiplas, seguidos pelos sujeitos submetidos ao Modelo Oposições Máximas Modificado. A aplicação dos modelos terapêuticos foi eficaz no tratamento dos sujeitos com DF, pois a maioria acrescentou sons em seus sistemas fonológicos, assim como apresentaram generalização a outras posições na palavra.

A generalização a outras posições na palavra ocorreu para todos os modelos estudados, todavia, os maiores percentuais de aumento obtidos foram nos Modelos ABAB-Retirada e Provas Múltiplas seguido de Oposições Máximas Modificado no grupo com desvio severo; nos Modelos Oposições Máximas Modificado, seguido do ABAB-Retirada e Provas Múltiplas, no grupo com desvio moderadosevero; no Modelo Oposições Máximas Modificado, no grupo com desvio médio-moderado; e nos Modelos Oposições Máximas Modificado e ABABRetirada e Provas Múltiplas; no grupo com desvio médio. 


\section{ABSTRACT}

Purpose: to analyze the efficacy in the three models of therapy with phonological basement in subjects with different degrees of phonological disorder severity. Methods: the subjects in this research were 21 children, eleven males and ten females, with age mean: 5:7. Speech data were analyzed through the performance of the phonological assessment. After the phonological assessment, the percentage of correct consonants proposed by Shriberg \& Kwiatkowski (1982) was calculated and the subjects were then classified in the severity degrees regarding the phonological disorder in: severe, moderatesevere, mild-moderate and mild disorder. Six0 subjects were submitted to the Modified Cycles Model, 8 to the ABAB-Withdrawal and Multiple Probes Model and 7 to the Modified Maximum Opposition Model. Making the comparison the generalization to other position in the word among three models of therapy within each degree of phonological disorder severity. Results: the generalization to other position in the word occurred in all the studied models, however, ABAB-Withdrawal and Multiple Probes Model were more effective to the group with severe disorder; the Modified Maximum Opposition Model to the group with moderate-severe and mild-moderate disorder; and the Modified Maximum Opposition and the ABAB-Withdrawal and Multiple Probes Models to the group with mild disorder. Conclusion: all the subjects showed gains in the phonological system and relation to generalization of the other positions in the word, but the ABAB-Withdrawal and Multiple Probes and Modified Maximum Opposition Models were more effective.

KEYWORDS: Speech Therapy; Generalization; Speech; Speech Disorders

\section{REFERÊNCIAS}

1. Mota HB, Keske-Soares M, Bagetti T, Ceron MI, Filha Melo MGC. Análise comparativa da eficiência de três diferentes modelos de terapia fonológica. Pró-Fono. 2007; 19(1):67-74.

2. Lamprecht RR. Sobre os desvios fonológicos. In: Lamprecht RR. Aquisição fonológica do Português. Perfil de desenvolvimento e subsídios para a terapia. Porto Alegre: Artmed; 2004. p. 193-212.

3. Wertzner HF, Pagan LO, Galea DES, Papp ACCS. Características fonológicas de crianças com transtorno fonológico com e sem histórico de otite média. Rev Soc Bras Fonoaudiol. 2007; 12(1):41-7.

4. Wertzner HF, Amaro L, Teramoto SS. Gravidade do distúrbio fonológico: julgamento perceptivo e porcentagem de consoantes corretas. Pró-Fono. 2005; 17(2):185-94.

5. Spíndola RA, Payão LMC, Bandini HHM. Abordagem fonoaudiológica em desvios fonológicos fundamentada na hierarquia dos traços distintivos e na consciência fonológica. Rev CEFAC. 2007; 9(2):180-9.

6. Keske-Soares M, Brancalioni AR, Marini C, Pagliarin KC, Ceron MI. Eficácia da terapia para desvios fonológicos com diferentes modelos terapêuticos. Pró-Fono. 2008; 20(3):153-8.

7. Ceron MI, Keske-Soares M. Terapia fonológica: a generalização a itens não utilizados no tratamento (outras palavras). Rev CEFAC. 2007; 9(4):453-60.
8. Ceron MI, Keske-Soares M. Terapia fonológica: a generalização dentro de uma classe de sons e para outras classes de sons. Rev CEFAC. 2008; 10(3):311-20.

9. Williams AL. Assessment, target selection, and intervention: dynamic interactions within a systemic perspective. Topics Lang Disord. 2005; 25(3):231-42.

10. Williams AL. A systematic perspective for assessment and intervention: a case study. Adv Speech Lang Pathol. 2006; 8(3):245-56.

11. Mota HB, Bagetti T, Keske-Soares M, Pereira LF. A generalização baseada nas relações implicacionais em sujeitos submetidos à terapia fonológica. Pró-Fono. 2005; 17(1):99-110.

12. Tyler AA, Edwards ML, Saxman JH. Clinical application of two phonologically based treatment procedures. J Speech Hear Disord. 1987; 52(4): 393-409.

13. Tyler AA, Figursky GR. Phonetic inventory changes after treating distinctions along an implicational hierarchy. Clin Linguist Phon. 1994; 8:91-108.

14. Bagetti T, Mota HB, Keske-Soares M. Modelo de Oposições Máximas Modificado: uma proposta de tratamento para o desvio fonológico. Rev Soc Bras Fonoaudiol. 2005; 10(1):36-42.

15. Mota HB. Fonologia: Intervenção. In: Ferreira LP, Befi-Lopes DM, Limonge SCO. Tratado de Fonoaudiologia. São Paulo: Roca; 2004. p. 787-814. 
16. Mota HB, Pereira LF. A generalização na terapia dos desvios fonológicos: experiência com duas crianças. Pró-Fono. 2001; 13(2):141-6.

17. Mota HB, Bagetti T, Keske-Soares M, Pereira LF. A generalização em sujeitos com desvio fonológico médio-moderado tratados pelo modelo de oposições máximas. Rev Soc Bras Fonoaudiol. 2004; 9:102-11.

18. Tyler AA. Commentary on "Treatment decisions for children with speech-sound disorders": revisiting the past in EBP. Lang Speech Hear Serv Schools. 2006; 37(4):280-3.

19. Gierut JA, Dale RA. Comparability of lexical corpora: word frequency in phonological generalization. Clin Linguist Phon. 2007; 21(6):423-33.

20. Powell TW, Elbert M, Dinnsen DA. Stimulability as a factor in the phonological generalization of misarticulating preschool children. J Speech Hear Res. 1991; 34(6):1318-28.

21. Crosbie S, Holm A, Dodd B. Intervention for children with severe speech disorder: a comparison of two approaches. Int $\mathrm{J}$ Lang Commun Disord. 2005; 40(4):467-91.

22. Blanco AP. A generalização no Modelo de Ciclos Modificado em pacientes com diferentes graus de severidade de desvio fonológico [dissertação]. Santa Maria (RS): Universidade Federal de Santa Maria; 2003.
23. Barberena L. A generalização obtida pelo Modelo "ABAB - Retirada e Provas Múltiplas" em diferentes graus de severidade do desvio fonológico [dissertação]. Santa Maria (RS): Universidade Federal de Santa Maria; 2005.

24. Donicht G. A generalização obtida a partir do tratamento como os róticos em dois modelos de terapia fonoaudiológica para crianças com desvios fonológicos [monografia]. Santa Maria (RS): Universidade Federal de Santa Maria; 2005.

25. Bagetti T. Mudanças fonológicas em sujeitos com diferentes graus de severidade do desvio fonológico tratados pelo Modelo de Oposições Máximas Modificado [dissertação]. Santa Maria (RS): Universidade Federal de Santa Maria; 2005.

26. Elbert M, Gierut JA. Handbook of clinical phonology. London: Taylor \& Francis; 1986.

27. Yavas M, Hernandorena CLM, Lamprecht RR. Avaliação fonológica da criança: reeducação e terapia. Porto Alegre: Artes Médicas; 1991.

28. Bernhardt B. Developmental implications of nonlinear phonological theory. Clin Linguist Phon. 1992; 6(4):259-81.

29. Shriberg LD, Kwiatkowski J. Phonological disorders I: a diagnostic classification system. J Speech Hear Disord. 1982; 47(3):226-41.

RECEBIDO EM: 15/08/2007

ACEITO EM: 11/07/2008

Endereço para correspondência:

Marizete Ilha Ceron

Rua Bentevi, 215

Santa Maria - RS

CEP: 97035-130

E-mail: marizeteceron@ hotmail.com 